\title{
Self-management of Lambda-Connections in Optical Networks
}

\author{
Tiago Fioreze and Aiko Pras \\ University of Twente \\ Design and Analysis of Communication Systems (DACS) \\ Enschede, The Netherlands
}

\begin{abstract}
This paper presents a new idea for the management of lambda-connections in optical networks. The idea consists of making multi-service optical switches responsible for automatically detecting IP flows at the packet-level, creating lambda-connections for them, and moving them to the optical-level. In addition to that, they are also in charge of tearing down the connections when no longer needed. This new idea is the result of 1 year of research work at the University of Twente (UT) and it is aimed at resulting in a Ph.D. thesis by the end of 4 years of Ph.D. research.
\end{abstract}

\section{Introduction}

Optical networks can send vast amounts of data (IP flows) through lambdaconnections. These connections are established through multi-service optical switches, which have the capability to perform forwarding decisions at different levels in the protocol stack. As a result, long and big IP flows (elephant flows) could be moved from the packet-level to the optical-level. This move could result in a better QoS for both elephant flows and remaining IP flows: the former would have no jitter and plenty of bandwidth at the optical-level; the latter would be better served due to the off-load of elephant flows.

The detection of IP flows and the management of lambda-connections are important tasks to achieve the desired move. Two approaches are currently used for that 1]: conventional management and GMPLS signaling. The former is characterized by a centralized management entity (e.g., human manager or an automated management process) that is in charge of establishing lambda-connections and deciding which IP flows should be moved to the optical-level. In contrast, the latter is characterized by the fact that optical switches coordinate the creation of lambda-connections among themselves. The decision which IP flows will be moved to the optical level however should be taken by a centralized management entity, or by the entities responsible for the generation of the data flow.

However, there are several problems using these approaches. Both approaches require human interaction to detect flows and manage lambda-connections. This interaction may be slow, since humans need time to perform those tasks, and it is also error prone. For instance, IP flows eligible to lambda-connections may not be detected by network managers. 
It is interesting to mention that the research work presented in this paper has been developed within the context of the SURFnet GigaPort Next Generation (Gigaport-NG) Research on Networking (RoN) project 22. This work has also the support of the EMANICS community [3], more specifically the collaboration of the INRIA Lorraine institute [4].

The remaining of this paper is structured as follows. Section 2 introduces our idea on self-management of lambda-connections in optical networks. Then the research questions and the approaches to answer those questions are introduced in section 3. Finally, conclusions and future plans are drawn in section 4.

\section{Proposed Idea}

This section introduces what self-management of lambda-connections stands for. Self-management of lambda-connections consists of an automatic cooperation between the IP and optical domain in order to create lambda-connections for IP flows. The network domain is in charge of detecting IP flows to be transferred over lambda-connections and signalizing the optical domain about the existence of these IP flows. On its turn, the optical domain is in charge of creating lambdaconnections for IP flows and releasing them when no longer needed. Figure 1 depicts how our proposed idea would look like.

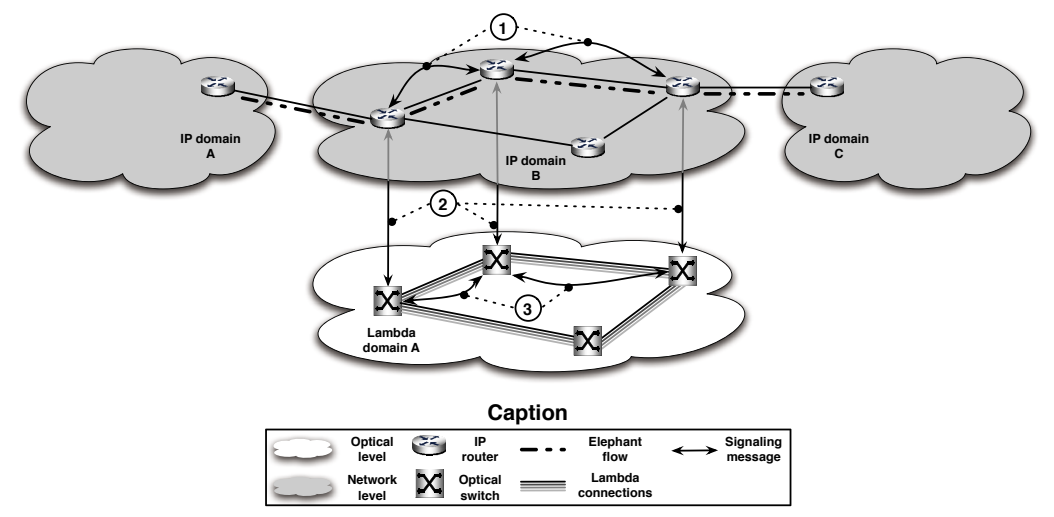

Fig. 1. Self-management of lambda-connection in optical networks

In Figure 1 IP routers located at IP domain B detect one elephant flow transiting between IP domains A and C. They start then talking to one another in order to confirm the existence of the detected elephant flow (step 1). When confirmed the existence, the IP routers signalize the optical switches in lambda domain A (step 2). The optical switches coordinate among themselves in order to create a dedicated lambda-connection to the detected elephant IP flow (step 3). From that point on, the elephant flow is switched at the optical level.

Further information about our idea on self-management can be found at [5]. 


\section{Research Questions and Proposed Approaches}

Since self-management of lambda-connections does not exist in current optical networks, it has to be designed. However, before a self-management architecture is designed, solutions for several research questions should be found. The source of these questions is the introduction of our new idea for managing optical networks. The following questions should therefore be considered and answered:

1. What are the pros $\&$ cons of self-management of lambda-connections compared to current approaches?

2. How can self-management be implemented?

This question can better be refined into a number of subquestions:

(a) What are the requirements to a flow being eligible to a lambda-connection?

(b) How can optical switches automatically detect IP flows?

(c) How can the establishment of lambda-connections be?

(d) How can IP flows be monitored over lambda-connections?

(e) How can the releasing of lambda-connections be?

Research question 1 aims at comparing our idea with the current management approaches: conventional management and GMPLS signaling. The approach to be taken to answer this question consists of finding out the proper criteria for this comparison. In order to do that, study of the literature and interview with professionals in the network management area will be done.

On its turn, research question 2 envisages explaining how self-management can be implemented. Each subquestion of this question is related to different stages of our implementation. Research question 2.(a) aims at presenting which requirements a IP flow should satisfy to be transferred over a lambda-connection. Some requirements have already been found due to the research work performed in the GigaPort-NG RoN project (see at [6]). The requirements are related to the duration and size of a flow, and as well network policies (that can be different at different network domains). A flow should therefore last more than the time to a lambda-connection is established, have the minimum size for a lambda-connection, and satisfy network policies. Further requirements will be investigated by using different optical networks (e.g., SURFnet6) as case study and by studying the literature as well.

Research question 2.(b) will show how the optical switches should cooperate to detect flows at the packet-level. Inter- and intra-domains will be considered in this question. The approach will consist of creating evaluation scenarios where the number of optical switches in charge of detecting IP flows will vary according to their location in the network domain. The scenarios will consider optical switches located at the edge, core or everywhere in the domain. The evaluation will likely be done by means of simulation tools (e.g., NS2 [7]) or by mathematical theory (e.g., Stochastic Petri nets).

Research question 2.(d) will investigate how IP flows can be monitored when carried over lambda-connections. Two initial ideas are considered: monitoring at optical-level or monitoring at packet-level. The former requires a literature 
study to check if there are technologies available for monitoring traffic at the optical-level. The latter can be done by monitoring the traffic at the end-points of the optical connections at the packet-level. In this case an evaluation will be performed to check if both end-points are required to monitor IP flows or only one of them. This evaluation will probable be done by using simulation tools.

Last but not least, research questions 2.(c) and 2.(e) aim at showing how the establishment and releasing of lambda-connections can be performed. The approach to answer these questions will focus on the study of the literature. It is worth to point out that some technologies existing in the Generalized Multiprotocol Label Switching (GMPLS) architecture 8 8 already do that, and they can therefore be considered strong candidates to be used in our research work.

Some of the results obtained so far in our research can be found at [9].

\section{Conclusions}

This paper presented the long-term goal that is intended to be achieved after a Ph.D. period of 4 years. It also showed the investigated problem, the research questions and approaches to be used to answer those questions. The next short-term goal of this research work is going to be the definition of our self-management architecture, where the involved components and their interaction are going to be defined. We would like to thank SURFnet for allowing us to perform measurements on their network. This paper was supported in part by the EC IST-EMANICS Network of Excellence (\#26854).

\section{References}

1. Bernstein, G., Rajagopalan, B., Saha, D.: Optical Network Control: Architecture, Protocols, and Standards. Addison Wesley Publishers, London, UK (2003)

2. GigaPort: GigaPort homepage, Available in (2007), http://www.surfnet.nl/info/en

3. EMANICS: European Network of Excellence for the Management of Internet Technologies and Complex Services (EMANICS) (2007) Available in: http://www.emanics.org/

4. INRIA-Lorraine: INRIA Lorraine Research Unit (2007) Available in: http://www.inria.fr/inria/organigramme/fiche_ur-lor.en.html

5. Fioreze, T., Pras, A.: Using self-management for establishing light paths in optical networks: an overview. In: Poster session proceedings of the 12th EUNICE Open European Summer School 2006 (EUNICE 2006) 17-20 ( 2006)

6. Fioreze, T.: D1.1.2 Requirements for lambda-connection management. Internal report GigaPort-NG RoN project (2006) Available in : http://wwwhome.cs.utwente.nl/ fiorezet/ron/Deliverable1.1.2(2006).pdf

7. The Network Simulator ns-2: The Network Simulator ns-2 homepage (2007) Availabe in: http://www.isi.edu/nsnam/ns/

8. Mannie, E.: Generalized Multi-Protocol Label Switching (GMPLS) Architecture Request for Comments: 3495 (RFC 3495) (2004)

9. Fioreze, T., Wolbers, M.O., Meent, R., Pras, A.: Finding elephant flows for optical networks. In: Application session proceedings of the 10th IFIP/IEEE International Symposium on Integrated Network Management (to appear) (IM 2007) 